\title{
Response to 'Storm forecasting: additional lessons from the CD28 superagonist TGN 1412 trial'
}

\section{Thomas Hünig}

In their correspondence piece on my recently published Comment article (The storm has cleared: lessons from the CD28 superagonist TGN1412 trial. Nature Rev. Immunol. 12, 317-318 (2012)) ${ }^{1}$, Horvath et al. (Storm forecasting: additional lessons from the CD28 superagonist TGN1412 trial. Nature Rev. Immunol. 31 Aug 2012 (doi:10.1038/nri3192-c1)) $)^{2}$ do not consider the recent evidence I discussed regarding the failure of pre-trial experimentation to predict the TGN1412-induced cytokinerelease syndrome (CRS). Instead, their critique focuses on the interpretation of the preclinical data available in 2006 for the design of the ill-fated trial, including the selection of the cynomolgus primate model for toxicity testing.

I wish to clarify that my Comment article did not deal with these issues. Rather, I pointed out that the new results published during the past few years on species-specific differences in CD28 superagonist responses in vivo and on the pitfalls of in vitro assays have provided a plausible explanation as to why the preclinical data available in 2006 led to a misprojection of the risk for toxicity with TGN1412 in humans. Indeed, it is now clear why rodents do not respond to CD28 superagonist treatment with systemic cytokine release $\mathrm{e}^{3}$, why cynomolgus macaques were not a relevant primate species for toxicity testing ${ }^{4}$, and why conventional human peripheral blood mononuclear cell (PBMC) assays do not reveal a cytokine burst in response to soluble TGN1412, as they do with OKT3, the monoclonal antibody used for comparison both by myself ${ }^{5}$ and by Horvath et al.

It is correct that $\mathrm{T}$ cell activation, in particular interleukin-2 (IL-2) production, is part of the intended mechanism of action of CD28 superagonists. This mechanism results in the expansion and functional activation of regulatory $\mathrm{T}$ cell populations (for which IL-2 is required in a non-redundant manner) ${ }^{6}$, and the expansion of $\mathrm{T}$ cell populations to accelerate recovery from lymphopenia ${ }^{7}$. Obviously, an IL-2 burst such as that experienced in the human volunteers was as unintended as it was unanticipated. Eastwood et al. ${ }^{4}$ and Römer et al. ${ }^{5}$ have since shown that the source of this IL-2 burst is effector memory $\mathrm{CD}^{+} \mathrm{T}$ cells, which, as discussed in the Comment article, are frequent in adult humans but rare in 'clean' laboratory rodents. The absence of CD28 expression on cynomolgus monkey effector memory $\mathrm{CD} 4^{+}$ $\mathrm{T}$ cells now provides a convincing reason why an increase in circulating $\mathrm{T}$ cell numbers, but not a cytokine burst, was observed in this preclinical model for TGN1412 (REF. 4).

With regard to the effects of TGN1412 on the human immune system itself, Horvath and colleagues quote a preclinical data set published shortly after the trial from which they would have predicted the CRS experienced by the volunteers. In this study by Legrand et al. ${ }^{8}$, mice with a human haematopoietic stem cell-derived immune system (HIS mice) were treated with the murine TGN1412 precursor 5.11A1. Although no acute toxicity was observed and regulatory $\mathrm{T}$ cells actually transiently accumulated in the periphery, the depletion of conventional human T cells in antibody-treated HIS mice is seen by Horvath et al. as evidence for 'superactivation', indicative of a threatening CRS. The authors of the study discuss three possibilities for the mechanism of T cell depletion in that system: antibody-dependent cell-mediated cytotoxicity (ADCC) and complement-mediated depletion (because, in contrast to the IgG4 antibody TGN1412, 5.11A1 is of the IgG1 subclass), and sensitivity of recent thymic emigrants to the induction of cell death by activating signals. Whatever the mechanism, it was most likely unrelated to the acute toxicity observed during the human trial. Thus, as mentioned above, the cytokine burst in humans was mediated by effector memory CD $4^{+} \mathrm{T}$ cells, whereas the HIS mice contained only recent thymic emigrants, which do not respond with such effector functions. Furthermore, systemic tumour necrosis factor release would have induced apoptosis in the thymocytes of the HIS mice; however, thymocyte numbers actually increased in response to the $\mathrm{CD} 28$ superagonist. Most importantly, however, Horvath et al. describe these effects as occurring at "as little as $\sim 0.3 \mathrm{mg}$ per kg" when, in fact, the doses that were used by Legrand et al. are reported as mg per mouse, which translates into a 50 -fold higher value (15 mg per kg, 150-fold more than during the human trial). At the lowest dose tested ( $0.03 \mathrm{mg}$ per mouse or $1.5 \mathrm{mg}$ per $\mathrm{kg}$ ), which was still 15-fold higher than the one used during the first-in-human trial, no effects were observed at all. Accordingly, Horvath et al. should rather quote this study as predicting a misleading safety margin over the first-in-human dose applied.

Coming back to the topic of my Comment article, the identification of the deficiencies of preclinical in vitro and in vivo systems in modelling the TGN1412-triggered CRS not only reminds us to more critically interpret results obtained in those specific assays, but also to be prepared for unexpected pitfalls of complex biological test systems in general.

\section{Thomas Hünig is at the Institute for Virology and Immunobiology, Versbacher Strasse 7, D-97078 Würzburg, Germany. e-mail: huenig@vim.uni-wuerzburg.de doi: 10.1038/nri3192-c2 Published online 31 August 2012}

1. Hunig, T. The storm has cleared: lessons from the CD28 superagonist TGN 1412 trial. Nature Rev. Immunol. 12, 317-318 (2012).

2. Horvath, C. et al. Storm forecasting: additional lessons from the CD28 superagonist TGN 1412 trial. Nature Rev. Immunol. 31 Aug 2012 (doi:10.1038/nri3192-c1).

3. Gogishvili, T. et al. Rapid regulatory T-cell response prevents cytokine storm in CD28 superagonist treated mice. PLOS ONE 4, e4643 (2009).

4. Eastwood, D. et al. Monoclonal antibody TGN1412 trial failure explained by species differences in CD28 expression on $\mathrm{CD}^{+}{ }^{+}$effector memory T-cells. Br. J. Pharmacol. 161, 512-526 (2010).

5. Romer, P. S. et al. Preculture of PBMCs at high cell density increases sensitivity of T-cell responses, revealing cytokine release by $\mathrm{CD} 28$ superagonist TGN1412. Blood 118, 6772-6782 (2011).

6. Hunig, T. Manipulation of regulatory T-cell number and function with CD28-specific monoclonal antibodies. Adv. Immunol. 95, 111-148 (2007).

7. Elflein, K., Rodriguez-Palmero, M., Kerkau, T. \& Hunig, T. Rapid recovery from T lymphopenia by CD28 superagonist therapy. Blood 102, 1764-1770 (2003).

8. Legrand, N. et al. Transient accumulation of human mature thymocytes and regulatory T cells with CD28 superagonist in "human immune system" Rag2 ${ }^{-1}$ $\gamma^{-1-}$ mice. Blood 108, 238-245 (2006).

Competing interests statement

The author declares competing financial interests: see web version for details. 